\title{
Existence of Periodic Solutions for Nonlinear Fully Third-Order Differential Equations
}

\author{
Yunfei Zhang and Minghe Pei \\ Department of Mathematics, Beihua University, Jilin City 132013, China \\ Correspondence should be addressed to Minghe Pei; peiminghe@163.com
}

Received 16 January 2020; Accepted 12 March 2020; Published 6 April 2020

Academic Editor: Lars E. Persson

Copyright (C) 2020 Yunfei Zhang and Minghe Pei. This is an open access article distributed under the Creative Commons Attribution License, which permits unrestricted use, distribution, and reproduction in any medium, provided the original work is properly cited.

In this paper, we study the existence of periodic solutions to nonlinear fully third-order differential equation $x^{\prime \prime \prime}+f\left(t, x, x^{\prime}, x^{\prime \prime}\right)=$ $0, t \in \mathbb{R}:=(-\infty, \infty)$, where $f: \mathbb{R}^{4} \longrightarrow \mathbb{R}$ is continuous and $T$-periodic in $t$. By using the topological transversality method together with the barrier strip technique, we obtain new existence results of periodic solutions to the above equation without growth restrictions on the nonlinearity. Meanwhile, as applications, an example is given to demonstrate our results.

\section{Introduction}

In this paper, we consider the existence of periodic solutions for nonlinear fully third-order differential equation

$$
x^{\prime \prime \prime}(t)+f\left(t, x(t), x^{\prime}(t), x^{\prime \prime}(t)\right)=0, \quad t \in \mathbb{R},
$$

where $f: \mathbb{R}^{4} \longrightarrow \mathbb{R}$ is continuous and $T$-periodic with respect to $t$.

The third-order periodic problem arises in many areas of applied mathematics and physics, and so it has been extensively studied by many authors via various methods, for instance, see [1-22] and the references therein. Among a substantial number of works, we mention that the upper and lower solutions method is used in $[5,6,15,16]$, Leray-Schauder continuation theorem is used in $[1,10,20,21]$, Leray-Schauder degree theory or the Schauder-fixed-point theorem is used in $[10,12,13,18]$, Mawhin coincidence degree theory is used in $[2,3,8,17]$, and fixed-point theorem in cone or fixed-point index theory is used in $[7,9,11,19$, 22 ]. However, to the best of our knowledge, there is no work that refers to periodic solutions of equation (1) using the topological transversality method.

Recently, Kelevedjiev and Todorov [23] have used the topological transversality method and barrier strip technique to study various third-order two-point boundary value problems. But, they did not consider the third-order periodic boundary value problem.

Motivated and inspired by the aforementioned works, the aim of this paper is to establish new existence results of periodic solutions to equation (1) by using the topological transversality method together with barrier strip technique. It is worth mentioning that our results do not need any growth restrictions on the nonlinearity. In addition, compared with the corresponding ones in the known literature, the barrier strip technique we use to estimate a prior bounds of periodic solutions is essentially new.

This work is organized as follows. In Section 2, we first introduce some notations and lemmas and then estimate a prior bounds of periodic solutions of equation (1) by using barrier strip technique. Finally, by using the topological transversality method, we establish the existence results of periodic solutions to equation (1). As applications of our main results, an example is given in the last section.

\section{Main Results}

Throughout this section, the following assumptions are used:

$\left(H_{0}\right)$ : there exists $M>0$ such that, for any $T$-periodic function $x \in C^{2}(\mathbb{R})$, 


$$
(\operatorname{sign} x(t)) \cdot \int_{0}^{T} f\left(t, x(t), x^{\prime}(t), x^{\prime \prime}(t)\right) \mathrm{d} t>0, \quad \text { if } \min _{t \in[0, T]}|x(t)|>M
$$

$\left(H_{0}^{\prime}\right)$ : there exists $M>0$ such that, for any $T$-periodic function $x \in C^{2}(\mathbb{R})$,

$$
(\operatorname{sign} x(t)) \cdot \int_{0}^{T} f\left(t, x(t), x^{\prime}(t), x^{\prime \prime}(t)\right) \mathrm{d} t<0, \quad \text { if } \min _{t \in[0, T]}|x(t)|>M
$$

$\left(H_{1}\right)$ : there exists $M_{1}>0$ such that

$y f(t, x, y, 0)<0, \quad \forall(t, x) \in[0, T] \times \mathbb{R},|y|>M_{1}$.

$\left(H_{2}\right)$ : there are constants $L_{i}, i=1,2,3,4$ with $L_{2}>L_{1}>0$ and $L_{3}<L_{4}<0$ such that $f(t, x, y, z)$ does not change its sign for $(t, x, y, z) \in D_{1}$ and for $(t, x, y, z) \in D_{2}$, respectively, here

$$
\begin{aligned}
& D_{1}=[0, T] \times\left[-M_{0}, M_{0}\right] \times\left[-M_{1}, M_{1}\right] \times\left[L_{1}, L_{2}\right], \\
& D_{2}=[0, T] \times\left[-M_{0}, M_{0}\right] \times\left[-M_{1}, M_{1}\right] \times\left[L_{3}, L_{4}\right],
\end{aligned}
$$

and $M_{0}=M+T M_{1}$.

Let us introduce some notations of the topological transversality method for the convenience of the reader. Let $U$ be a convex subset of a Banach space $Y$ and $\Omega \subset U$ be open in $U$. Denote by $H_{\partial \Omega}(\bar{\Omega}, U)$ the set of compact operators $F: \bar{\Omega} \longrightarrow U$ which are fixed-point-free on $\partial \Omega$. We say that $F \in H_{\partial \Omega}(\bar{\Omega}, U)$ is essential if every operator in $H_{\partial \Omega}(\bar{\Omega}, U)$ which agrees with $F$ on $\partial \Omega$ has a fixed point in $\Omega$.

The next two lemmas can be found in [24].

Lemma 1. If $p \in \Omega$ and $F \in H_{\partial \Omega}(\bar{\Omega}, U)$ is a constant operator, $F(y)=p$ for $y \in \bar{\Omega}$ then is essential.

\section{Lemma 2. Let}

(i) $F \in H_{\partial \Omega}(\bar{\Omega}, U)$ be essential

(ii) $H: \bar{\Omega} \times[0,1] \longrightarrow U$ be a compact homotopy, $H(\cdot, 0)=F$, and $H(y, \lambda) \neq y$ for $y \in \partial \Omega$ and $\lambda \in[0,1]$

Then, $H(\cdot, 1)$ is essential, and therefore, it has a fixed point in $\Omega$.

We note that the existence of $T$-periodic solutions for equation (1) is equivalent to the solvability of the following third-order periodic boundary value problem (for short PBVP):

$$
\left\{\begin{array}{l}
x^{\prime \prime \prime}(t)+f\left(t, x(t), x^{\prime}(t), x^{\prime \prime}(t)\right)=0, \quad 0 \leq t \leq T, \\
x(0)=x(T), \\
x^{\prime}(0)=x^{\prime}(T), \\
x^{\prime \prime}(0)=x^{\prime \prime}(T),
\end{array}\right.
$$

in $C^{2}[0, T]$.

We first consider the family of following PBVPs:

$$
\begin{aligned}
x^{\prime \prime \prime}(t)+\lambda f\left(t, x(t), x^{\prime}(t)\right. & \left., x^{\prime \prime}(t)\right)=0, \quad 0 \leq t \leq T, \\
x(0) & =x(T), \\
x^{\prime}(0) & =x^{\prime}(T), \\
x^{\prime \prime}(0) & =x^{\prime \prime}(T),
\end{aligned}
$$

where $\lambda \in(0,1]$.

A priori bounds for the solutions of PBVP (7), (8) are presented in the following lemmas.

Lemma 3. Suppose that $\left(H_{0}\right)\left(\right.$ or $\left.\left(H_{0}^{\prime}\right)\right)$ and $\left(H_{1}\right)$ hold. Let $x(t)$ be a solution of PBVPs (7) and (8) for some $\lambda \in(0,1]$. Then,

$$
\begin{aligned}
& |x(t)| \leq M_{0}, \quad \forall t \in[0, T], \\
& |x \prime(t)| \leq M_{0}, \quad \forall t \in[0, T] .
\end{aligned}
$$

Proof. At first, we show that (10) holds. Indeed, suppose on the contrary that there exist $t_{1} \in[0, T]$ such that $\left|x^{\prime}\left(t_{1}\right)\right|>M_{1}$. We may assume that $x^{\prime}\left(t_{1}\right)>M_{1}$. Let $t_{2} \in[0, T]$ be such that

$$
x^{\prime}\left(t_{2}\right)=\max _{t \in[0, T]} x^{\prime}(t)>M_{1} .
$$

Without loss of generality, we assume that $t_{2} \in(0, T)$; then, $x^{\prime \prime}\left(t_{2}\right)=0$ and $x^{\prime \prime \prime}\left(t_{2}\right) \leq 0$. It follows from condition $\left(H_{1}\right)$ that

$$
0 \geq x^{\prime}\left(t_{2}\right) x^{\prime \prime \prime}\left(t_{2}\right)=-x^{\prime}\left(t_{2}\right) \lambda f\left(t_{2}, x\left(t_{2}\right), x^{\prime}\left(t_{2}\right), 0\right)>0,
$$

which is a contradiction. This means that (10) holds.

Next, we prove that (9) holds. Indeed, integrating the equation in (7) from 0 to $T$, we obtain that

$$
\int_{0}^{T} f\left(t, x(t), x^{\prime}(t), x^{\prime \prime}(t)\right) \mathrm{d} t=0 .
$$

This together with the condition $\left(H_{0}\right)\left(\right.$ or $\left.\left(H_{0}^{\prime}\right)\right)$ implies that there exists $\xi \in[0, T]$ such that $|x(\xi)| \leq M$. It follows from (10) that, for $t \in[0, T]$, 


$$
|x(t)|=\left|x(\xi)+\int_{\xi}^{t} x^{\prime}(s) \mathrm{d} s\right| \leq M+\int_{0}^{T}\left|x^{\prime}(s)\right| \mathrm{d} s \leq M_{0}
$$

which means that (9) holds. This completes the proof of the lemma.

Lemma 4. Suppose that $\left(H_{0}\right)$ (or $\left.\left(H_{0}^{\prime}\right)\right),\left(H_{1}\right)$, and $\left(H_{2}\right)$ hold. Let $x(t)$ be a solution of PBVPs (7) and (8) for some $\lambda \in(0,1]$. Then,

$$
\left|x^{\prime \prime}(t)\right| \leq M_{2}:=\max \left\{L_{1},-L_{4}\right\}, \quad \forall t \in[0, T] .
$$

Proof. We estimate $x^{\prime \prime}(t)$ by using the barrier strip technique. From condition $\left(\mathrm{H}_{2}\right), f(t, x, y, z)$ does not change its sign for $(t, x, y, z) \in D_{1}$ and for $(t, x, y, z) \in D_{2}$, respectively. For the sake of certainty, without loss of generality, we assume that

$$
\begin{array}{ll}
f(t, x, y, z) \leq 0, & \text { on } D_{1}, \\
f(t, x, y, z) \leq 0, & \text { on } D_{2} .
\end{array}
$$

Notice that $x^{\prime}(0)=x^{\prime}(T)$, and from Rolle's mean value theorem, there exists $\eta \in(0, T)$ such that $x^{\prime \prime}(\eta)=0$. Let

$$
\begin{aligned}
& S_{0}=\left\{t \in[0, \eta]: L_{1}<x^{\prime \prime}(t) \leq L_{2}\right\}, \\
& S_{1}=\left\{t \in[0, \eta]: L_{3} \leq x^{\prime \prime}(t)<L_{4}\right\} .
\end{aligned}
$$

We now assert that the sets $S_{0}$ and $S_{1}$ are empty. We shall complete the proof in two steps.

Step 1. Show that $S_{0}=\varnothing$. Suppose on the contrary that there exist some $t_{0} \in S_{0}$. Then, $L_{1}<x^{\prime \prime}\left(t_{0}\right) \leq L_{2}$, and $t_{0} \in[0, \eta)$. Since $x^{\prime \prime}(t)$ is continuous on $[0, \eta]$, there exist $t_{0} \leq t_{1}<t_{2}<\eta$ such that

$$
\begin{gathered}
L_{1}<x^{\prime \prime}\left(t_{2}\right)<x^{\prime \prime}\left(t_{1}\right) \leq L_{2}, \\
x^{\prime \prime}\left(t_{2}\right) \leq x^{\prime \prime}(t) \leq x^{\prime \prime}\left(t_{1}\right), \quad \forall t \in\left[t_{1}, t_{2}\right],
\end{gathered}
$$

and so $\left[t_{1}, t_{2}\right] \subset S_{0}$. Consequently, from assumption (16), we have

$$
x^{\prime \prime \prime}(t)=-\lambda f\left(t, x(t), x^{\prime}(t), x^{\prime \prime}(t)\right) \geq 0, \quad \forall t \in\left[t_{1}, t_{2}\right] \subset S_{0},
$$

and thus,

$$
x^{\prime \prime}\left(t_{2}\right) \geq x^{\prime \prime}\left(t_{1}\right)
$$

which contradicts (18). This implies that $S_{0}=\varnothing$.

Step 2. Prove that $S_{1}=\varnothing$. By contradiction, assume that there exist some $t_{0}^{\prime} \in S_{1}$. Then, $L_{3} \leq x^{\prime \prime}\left(t_{0}^{\prime}\right)<L_{4}$, and $t_{0}^{\prime} \in[0, \eta)$. We now assert that

$$
x^{\prime \prime}(0) \geq L_{4} \text {. }
$$

Indeed, if $x^{\prime \prime}(0) \geq L_{4}$, then from the fact $x^{\prime \prime}(0)=x^{\prime \prime}(T)$, it follows that $x^{\prime \prime}(T) \geq L_{4}$. Notice that $x^{\prime \prime}(\eta)=0$, and it follows from the continuity of $x^{\prime \prime}(t)$ on $[\eta, T]$ that there exist $\eta<\eta_{1}<\eta_{2} \leq T$ such that

$$
\begin{aligned}
L_{3} & \leq x^{\prime \prime}\left(\eta_{2}\right)<x^{\prime \prime}\left(\eta_{1}\right)<L_{4}, \\
x^{\prime \prime}\left(\eta_{2}\right) & \leq x^{\prime \prime}(t) \leq x^{\prime \prime}\left(\eta_{1}\right), \quad \forall t \in\left[\eta_{1}, \eta_{2}\right] .
\end{aligned}
$$

Hence, from Lemma 3, we have

$$
\left(t, x(t), x \prime(t), x^{\prime \prime}(t)\right) \in D_{2}, \quad \forall t \in\left[\eta_{1}, \eta_{2}\right],
$$

and so from (16), it follows that

$$
x^{\prime \prime \prime}(t)=-\lambda f\left(t, x(t), x \prime(t), x^{\prime \prime}(t)\right) \geq 0, \quad \forall t \in\left[\eta_{1}, \eta_{2}\right] .
$$

Therefore,

$$
x^{\prime \prime}\left(\eta_{2}\right) \geq x^{\prime \prime}\left(\eta_{1}\right)
$$

which contradicts (22). This means that (21) holds.

Thus, from (21) and the continuity of $x^{\prime \prime}(t)$ on $[0, \eta]$, there exist $0<t_{1}^{\prime}<t_{2}^{\prime}<t_{0}^{\prime}$ such that

$$
\begin{aligned}
L_{3} & \leq x^{\prime \prime}\left(t_{2}^{\prime}\right)<x^{\prime \prime}\left(t_{1}^{\prime}\right)<L_{4}, \\
x^{\prime \prime}\left(t_{2}^{\prime}\right) & \leq x^{\prime \prime}(t) \leq x^{\prime \prime}\left(t_{1}^{\prime}\right), \quad \forall t \in\left[t_{1}^{\prime}, t_{2}^{\prime}\right],
\end{aligned}
$$

and thus, $\left[t_{1}^{\prime}, t_{2}^{\prime}\right] \subset S_{1}$. It follows from assumption (16) that

$$
x^{\prime \prime \prime}(t)=-\lambda f\left(t, x(t), x \prime(t), x^{\prime \prime}(t)\right) \geq 0, \quad \forall t \in\left[t_{1}^{\prime}, t_{2}^{\prime}\right] \subset S_{1},
$$

and hence,

$$
x^{\prime \prime}\left(t_{1}^{\prime}\right) \leq x^{\prime \prime}\left(t_{2}^{\prime}\right)
$$

which contradicts (26). This implies that $S_{1}=\varnothing$.

Therefore, by the facts that $x^{\prime \prime}(\eta)=0$ and the continuity of $x^{\prime \prime}(t)$ on $[0, \eta]$, we obtain

$$
L_{4} \leq x^{\prime \prime}(t) \leq L_{1}, \quad \forall t \in[0, \eta] .
$$

In particular, $L_{4} \leq x^{\prime \prime}(0) \leq L_{1}$. Notice $x^{\prime \prime}(0)=x^{\prime \prime}(T)$, and we have

$$
L_{4} \leq x^{\prime \prime}(T) \leq L_{1}
$$

We now let

$$
\begin{aligned}
& S_{2}=\left\{t \in[\eta, T]: L_{1}<x^{\prime \prime}(t) \leq L_{2}\right\}, \\
& S_{3}=\left\{t \in[\eta, T]: L_{3}<x^{\prime \prime}(t) \leq L_{4}\right\} .
\end{aligned}
$$

Notice that, from (30), using the similar arguments on $S_{0}=\varnothing$ and $S_{1}=\varnothing$, we can show that

$$
\begin{aligned}
& S_{2}=\varnothing, \\
& S_{3}=\varnothing .
\end{aligned}
$$

Hence,

$$
L_{4} \leq x^{\prime \prime}(t) \leq L_{1}, \quad \forall t \in[\eta, T] .
$$

From this, together with (29), it follows that

$$
L_{4} \leq x^{\prime \prime}(t) \leq L_{1}, \quad \forall t \in[0, T],
$$

which implies that 


$$
\left|x^{\prime \prime}(t)\right| \leq \max \left\{L_{1},-L_{4}\right\}=M_{2}, \quad \forall t \in[0, T] .
$$

This completes the proof of the lemma.

Now, denote $Y=C^{2}[0, T] \times \mathbb{R}$ the Banach space equipped with the norm $\|(x, r)\|=\|x\|_{\infty}+\|x \prime\|_{\infty}+$ $\left\|x^{\prime \prime}\right\|_{\infty}+|r|$. Set

$$
\begin{aligned}
U & =\{(x, r) \in Y: x(0)=x(T)=0, r \in \mathbb{R}\}, \\
\Omega & =\left\{(x, r) \in U:\|x\|_{\infty}<2 M_{0}+1,\|x \prime\|_{\infty}<M_{1}+1,\left\|x^{\prime \prime}\right\|_{\infty}\right. \\
& \left.<M_{2}+1,|r|<M_{0}+1\right\} .
\end{aligned}
$$

Then, $U$ is a closed and convex subset of $Y$, and $\Omega$ is an open subset of $U$.

We now give two lemmas which will be used in the proof of our main theorem.

Lemma 5. Suppose that $\left(H_{0}\right)$ holds. Let the operator $F_{1}: \bar{\Omega} \longrightarrow U$ be defined by

$$
F_{1}(x, r)=\left(0, r-\int_{0}^{T} f\left(\tau, x(\tau)+r, x^{\prime}(\tau), x^{\prime \prime}(\tau)\right) \mathrm{d} \tau\right) .
$$

Then, $F_{1}$ is essential.

Proof. Define $H: \bar{\Omega} \times[0,1] \longrightarrow U$ by

$$
H(x, r, \lambda)=\left(0, \lambda r-\lambda \int_{0}^{T} f\left(\tau, x(\tau)+r, x \prime(\tau), x^{\prime \prime}(\tau)\right) \mathrm{d} \tau\right) .
$$

Then, $H(\cdot, \cdot, 1)=F_{1}(\cdot, \cdot)$ and $H(x, r, 0)=(0,0) \in \Omega$ for $(x, r) \in \bar{\Omega}$. Thus, it follows from Lemma 1 that $H(x, r, 0)$ is essential. Meanwhile, by a standard argument, it is easy to show that $H(x, r, \lambda)$ is compact.

We now show that

$$
H(x, r, \lambda) \neq(x, r), \quad \forall(x, r) \in \partial \Omega, \lambda \in[0,1] .
$$

Obviously, $H(x, r, 0) \neq(x, r)$ for all $(x, r) \in \partial \Omega$. Suppose that $H\left(x_{0}, r_{0}, \lambda_{0}\right)=\left(x_{0}, r_{0}\right)$ for some $\left(x_{0}, r_{0}\right) \in \partial \Omega$ and $\lambda_{0} \in(0,1]$. Then, $x_{0}(t) \equiv 0$ on $[0, T]$, and so

$$
\int_{0}^{T} f\left(\tau, r_{0}, 0,0\right) \mathrm{d} \tau=\left(1-\frac{1}{\lambda}\right) r_{0} .
$$

Hence, from $\left(H_{0}\right)$, we can deduce that $-M \leq r_{0} \leq M$, which contradicts $\left(0, r_{0}\right) \in \partial \Omega$. This implies that (39) holds. Therefore, from Lemma $2, F_{1}(\cdot, \cdot)=H(\cdot, \cdot, 1)$ is essential. This completes the proof of the lemma.

Lemma 6. Suppose that $\left(H_{0}^{\prime}\right)$ holds. Let the operator $F_{2}: \bar{\Omega} \longrightarrow U$ be defined by

$$
F_{2}(x, r)=\left(0, r+\int_{0}^{T} f\left(\tau, x(\tau)+r, x \prime(\tau), x^{\prime \prime}(\tau)\right) \mathrm{d} \tau\right) .
$$

Then, $F_{2}$ is essential.

Proof. The proof is similar to the proof of Lemma 5 and hence is omitted.

Theorem 1. Suppose that $\left(H_{0}\right),\left(H_{1}\right)$, and $\left(H_{2}\right)$ hold. Then, equation (1) has at least one T-periodic solution $x=x(t)$ satisfying (9), (10), and (15).

Proof. At first, we define operator $A: \bar{\Omega} \times[0,1] \longrightarrow$ $C^{2}[0, T]$ by

$$
\begin{aligned}
A(x, r, \lambda)= & \lambda \int_{0}^{T} K(t, s)\left[\int_{0}^{s} f\left(\tau, x(\tau)+r, x \prime(\tau), x^{\prime \prime}(\tau)\right) \mathrm{d} \tau\right. \\
& \left.-\frac{1}{T} \int_{0}^{T}(T-\tau) f\left(\tau, x(\tau)+r, x \prime(\tau), x^{\prime \prime}(\tau)\right) \mathrm{d} \tau\right] \mathrm{d} s,
\end{aligned}
$$

where

$$
K(t, s)=\frac{1}{T} \begin{cases}t(T-s), & 0 \leq t \leq s \leq T ; \\ s(T-t), & 0 \leq s \leq t \leq T .\end{cases}
$$

It is easy to check that, for each $(x, r, \lambda) \in \bar{\Omega} \times[0,1]$, $A(x, r, \lambda)$ is the unique solution of the following boundary value problem:

$$
\left\{\begin{array}{l}
u^{\prime \prime \prime}+\lambda f\left(t, x(t)+r, x^{\prime}(t), x^{\prime \prime}(t)\right)=0, \quad t \in[0, T] \\
u(0)=u(T)=0 \\
u \prime(0)=u \prime(T) .
\end{array}\right.
$$

Furthermore, by a standard argument, it is easy to show that the operator $A(x, r, \lambda)$ is completely continuous.

We now define operator $G_{1}: \bar{\Omega} \times[0,1] \longrightarrow U$ by

$$
G_{1}(x, r, \lambda)=\left(A(x, r, \lambda), r-\int_{0}^{T} f\left(\tau, x(\tau)+r, x \prime(\tau), x^{\prime \prime}(\tau)\right) \mathrm{d} \tau\right) .
$$

Suppose that $\left(x_{1}, r_{1}\right)$ is a fixed point of $G_{1}(\cdot, \cdot, 1)$. Then,

$$
\begin{aligned}
x_{1}(t)= & \int_{0}^{T} K(t, s)\left[\int_{0}^{s} f\left(\tau, x_{1}(\tau)+r_{1}, x_{1}^{\prime}(\tau), x_{1}^{\prime \prime}(\tau)\right) \mathrm{d} \tau\right. \\
& \left.-\frac{1}{T} \int_{0}^{T}(T-\tau) f\left(\tau, x_{1}(\tau)+r_{1}, x_{1}^{\prime}(\tau), x_{1}^{\prime \prime}(\tau)\right) \mathrm{d} s\right], \\
& \int_{0}^{T} f\left(\tau, x_{1}(\tau)+r_{1}, x_{1}^{\prime}(\tau), x_{1}^{\prime \prime}(\tau)\right) \mathrm{d} \tau=0 .
\end{aligned}
$$

It follows that $x_{1}(0)=x_{1}(T)=0, x_{1}^{\prime}(0)=x_{1}^{\prime}(T)$, and 


$$
\begin{aligned}
-x_{1}^{\prime \prime}(t)= & \int_{0}^{t} f\left(\tau, x_{1}(\tau)+r_{1}, x_{1}^{\prime}(\tau), x_{1}^{\prime \prime}(\tau)\right) \mathrm{d} \tau \\
& -\frac{1}{T} \int_{0}^{T}(T-\tau) f\left(\tau, x_{1}(\tau)+r_{1}, x_{1}^{\prime}(\tau), x_{1}^{\prime \prime}(\tau)\right) \mathrm{d} \tau, \quad t \in[0, T],
\end{aligned}
$$

and thus, by (46),

$$
x_{1}^{\prime \prime}(0)=\frac{1}{T} \int_{0}^{T}(T-\tau) f\left(\tau, x_{1}(\tau)+r_{1}, x_{1}^{\prime}(\tau), x_{1}^{\prime \prime}(\tau)\right) \mathrm{d} \tau=x_{1}^{\prime \prime}(T) .
$$

Set $x_{2}(t)=x_{1}(t)+r_{1}$ for $t \in[0, T]$. It is easy to see that $x_{2}(t)$ is a solution of PBVP (6), and validity of (9), (10), and (15) now follows from Lemmas 3 and 4. Therefore, to prove the existence of solutions of PBVP (6) satisfying (9), (10), and (15), it is sufficient to show that the operator $G_{1}(\cdot, \cdot, 1)$ has at least one fixed point. Since $G_{1}(\cdot, \cdot, 0)=F_{1}(\cdot, \cdot)$ and $F_{1}$ is essential by Lemma 5 , for the existence of a fixed point of $G_{1}(\cdot, \cdot, 1)$, it is sufficient to verify (i) and (ii) of Lemma 2. Notice that operator $A$ is completely continuous, then operator $G_{1}$ is continuous, and also $G_{1}(\bar{\Omega} \times[0,1])$ is compact in $U$. Thus, (i) of Lemma 2 is satisfied. Let $G_{1}\left(x_{0}, r_{0}, \lambda_{0}\right)=$ $\left(x_{0}, r_{0}\right)$ for some $\left(x_{0}, r_{0}\right) \in \partial \Omega$ and $\lambda_{0} \in[0,1]$. If $\lambda_{0}=0$, then $\left(x_{0}, r_{0}\right) \notin \partial \Omega$, which has been proved in the proof of Lemma 5. Let $\lambda_{0} \in(0,1]$. Then,

$$
\begin{aligned}
x_{0}(t)= & \lambda_{0} \int_{0}^{T} K(t, s)\left[\int_{0}^{s} f\left(\tau, x_{0}(\tau)+r_{0}, x_{0}^{\prime}(\tau), x_{0}^{\prime \prime}(\tau)\right) \mathrm{d} \tau\right. \\
& \left.-\frac{1}{T} \int_{0}^{T}(T-\tau) f\left(\tau, x_{0}(\tau)+r_{0}, x_{0}^{\prime}(\tau), x_{0}^{\prime \prime}(\tau)\right) \mathrm{d} \tau\right] \mathrm{d} s, \\
& \int_{0}^{T} f\left(\tau, x_{0}(\tau)+r_{0}, x_{0}^{\prime}(\tau), x_{0}^{\prime \prime}(\tau)\right) \mathrm{d} \tau=0 .
\end{aligned}
$$

Hence,

$$
\begin{aligned}
-x_{0}^{\prime \prime}(t) & =\lambda_{0} f\left(t, x_{0}(t)+r_{0}, x_{0}^{\prime}(t), x_{0}^{\prime \prime}(t)\right), \quad t \in[0, T] \\
x_{0}(0) & =x_{0}(T)=0 \\
x_{0}^{\prime}(0) & =x_{0}^{\prime}
\end{aligned}
$$

and so

$$
x_{0}^{\prime \prime}(0)=\frac{1}{T} \int_{0}^{T}(T-\tau) f\left(\tau, x_{0}(\tau)+r_{0}, x_{0}^{\prime}(\tau), x_{0}^{\prime \prime}(\tau)\right) \mathrm{d} \tau=x_{0}^{\prime \prime}(T) .
$$

Set $x(t)=x_{0}(t)+r_{0}$ for $t \in[0, T]$. We can see that $x(t)$ is a solution of PBVPs (7) and (8) with $\lambda=\lambda_{0}$. It follows from Lemmas 4 and 5 that

$$
\begin{aligned}
\left\|x_{0}+r_{0}\right\|_{\infty} & =\|x\|_{\infty} \leq M_{0}, \\
\left\|x_{0}^{\prime}\right\|_{\infty} & =\left\|x^{\prime}\right\|_{\infty} \leq M_{1}<M_{1}+1, \\
\left\|x_{0}^{\prime \prime}\right\|_{\infty} & =\left\|x^{\prime \prime}\right\|_{\infty} \leq M_{2}<M_{2}+1 .
\end{aligned}
$$

Since $x_{0}(0)=0$, the first inequality of (52) yields

$$
\left|r_{0}\right| \leq M_{0}<M_{0}+1
$$

and thus,

$$
\left\|x_{0}\right\|_{\infty} \leq M_{0}+\left|r_{0}\right| \leq 2 M_{0}<2 M_{0}+1 \text {. }
$$

Hence, $\left(x_{0}, r_{0}\right) \notin \partial \Omega$, and so (ii) of Lemma 2 is verified. This completes the proof of the theorem.

Theorem 2. Suppose that $\left(H_{0}^{\prime}\right),\left(H_{1}\right)$, and $\left(H_{2}\right)$ hold. Then, equation (1) has at least one T-periodic solution $x=x(t)$ satisfying (9), (10), and (15).

Proof. The proof is similar as that for Theorem 1 except that

$$
G_{2}(x, r, \lambda):=\left(A(x, r, \lambda), r+\int_{0}^{T} f\left(\tau, x(\tau)+r, x \prime(\tau), x^{\prime \prime}(\tau)\right) \mathrm{d} \tau\right)
$$

and $F_{2}$ are used in place of $G_{1}(x, r, \lambda)$ and $F_{1}$, respectively, and hence is omitted. This completes the proof of the theorem.

\section{An Example}

In this section, we give an example to demonstrate the applications of the our results.

Example 1. Consider the nonlinear third-order differential equation

$$
x^{\prime \prime \prime}+\left(x^{\prime 2}+1\right) x^{\prime \prime}-\left(1-\frac{1}{2} \cos x\right) x^{\prime}+\frac{x-1}{x^{2}+1}=\sin t, \quad t \in \mathbb{R} .
$$

Let

$f(t, x, y, z)=\left(y^{2}+1\right) z-\left(1-\frac{1}{2} \cos x\right) y+\frac{x-1}{x^{2}+1}-\sin t$,

$$
(t, x, y, z) \in \mathbb{R}^{4} \text {. }
$$

Then, for any $2 \pi$-periodic function $x \in C^{2}(\mathbb{R})$, we have

$$
(\operatorname{sign} x(t)) \int_{0}^{2 \pi} f\left(t, x(t), x^{\prime}(t), x^{\prime \prime}(t)\right) \mathrm{d} t=(\operatorname{sign} x(t)) \int_{0}^{2 \pi} \frac{x(t)-1}{x^{2}(t)+1} \mathrm{~d} t>0, \quad \text { if } \min _{t \in[0,2 \pi]}|x(t)|>1:=M
$$


and so condition $\left(H_{0}\right)$ holds. Notice that

$$
\lim _{y \longrightarrow \pm \infty} y f(t, x, y, 0)=\lim _{y \longrightarrow \pm \infty} y\left(-\left(1-\frac{1}{2} \cos x\right) y+\frac{x-1}{x^{2}+1}-\sin t\right)=-\infty
$$

uniformly in $(t, x) \in[0,2 \pi] \times \mathbb{R}$; then, there exists $M_{1}>0$ such that

$$
y f(t, x, y, 0)<0, \quad \forall(t, x) \in[0,2 \pi] \times \mathbb{R},|y|>M_{1},
$$

that is, condition $\left(H_{1}\right)$ holds. In addition, since

$$
\lim _{z \longrightarrow \pm \infty} f(t, x, y, z)= \pm \infty \text {, }
$$

uniformly in $(t, x, y) \in[0,2 \pi] \times\left[-M_{0}, M_{0}\right] \times\left[-M_{1}, M_{1}\right]$, where $M_{0}=M+2 \pi M_{1}$, it follows that condition $\left(H_{2}\right)$ holds. Hence, by Theorem 1, third-order differential equation (56) has at least one $2 \pi$-periodic solution.

\section{Data Availability}

There are no data in this paper.

\section{Conflicts of Interest}

The authors declare that they have no conflicts of interest.

\section{Authors' Contributions}

All authors contributed equally to the writing of this paper. All authors read and approved the final manuscript.

\section{References}

[1] A. R. Aftabizadeh, J.-M. Xu, and C. P. Gupta, "Periodic boundary value problems for third order ordinary differential equations," Nonlinear Analysis: Theory, Methods \& Applications, vol. 14, no. 1, pp. 1-10, 1990.

[2] A. U. Afuwape, P. Omari, and F. Zanolin, "Nonlinear perturbations of differential operators with nontrivial kernel and applications to third-order periodic boundary value problems," Journal of Mathematical Analysis and Applications, vol. 143, no. 1, pp. 35-56, 1989.

[3] P. Amster, P. De Nápoli, and M. C. Mariani, "Periodic solutions of a resonant third-order equation," Nonlinear Analysis: Theory, Methods \& Applications, vol. 60, no. 3, pp. 399-410, 2005.

[4] S. R. Baslandze and I. T. Kiguradze, "On the unique solvability of a periodic boundary value problem for third-order linear differential equations," Differential Equations, vol. 42, no. 2, pp. 165-171, 2006.

[5] Z. Benbouziane, A. Boucherif, and S. M. Bouguima, "Existence result for impulsive third order periodic boundary value problems," Applied Mathematics and Computation, vol. 206, no. 2, pp. 728-737, 2008.

[6] A. Cabada, "The method of lower and upper solutions for third-order periodic boundary value problems," Journal of Mathematical Analysis and Applications, vol. 195, no. 2, pp. 568-589, 1995.

[7] J. Chu and Z. Zhou, "Positive solutions for singular non-linear third-order periodic boundary value problems," Nonlinear
Analysis: Theory, Methods \& Applications, vol. 64, no. 7, pp. 1528-1542, 2006.

[8] J. O. C. Ezeilo and M. N. Nkashama, "Resonant and nonresonant oscillations for some third order nonlinear ordinary differential equations," Nonlinear Analysis: Theory, Methods \& Applications, vol. 12, no. 10, pp. 1029-1046, 1988.

[9] Y. Feng, "On the existence and multiplicity of positive periodic solutions of a nonlinear third-order equation," Applied Mathematics Letters, vol. 22, no. 8, pp. 1220-1224, 2009.

[10] L. Kong, S. Wang, and J. Wang, "Positive solution of a singular nonlinear third-order periodic boundary value problem," Journal of Computational and Applied Mathematics, vol. 132, no. 2, pp. 247-253, 2001.

[11] Y. Li, "Positive periodic solutions for fully third-order ordinary differential equations," Computers \& Mathematics with Applications, vol. 59, no. 11, pp. 3464-3471, 2010.

[12] W. B. Liu, J. J. Zhang, and T. Y. Chen, "Anti-symmetric periodic solutions for the third order differential systems," Applied Mathematics Letters, vol. 22, no. 5, pp. 668-673, 2009.

[13] F. M. Minhós, "Periodic solutions for a third order differential equation under conditions on the potential," Portugaliae Mathematica, vol. 55, pp. 475-484, 1998.

[14] S. Mukhigulashvili, "On a periodic boundary value problem for third order linear functional differential equations," Nonlinear Analysis: Theory, Methods \& Applications, vol. 66, no. 2, pp. 527-535, 2007.

[15] J. J. Nieto, "Periodic solutions for third order ordinary differential equations," Commentationes Mathematicae Universitatis Carolinae, vol. 32, pp. 495-499, 1991.

[16] P. Omari and M. Trombetta, "Remarks on the lower and upper solutions method for second- and third-order periodic boundary value problems," Applied Mathematics and Computation, vol. 50, no. 1, pp. 1-21, 1992.

[17] I. Rachůnková, "Periodic boundary value problems for third order nonlinear differential equations," Mathematica Slovaca, vol. 41, pp. 241-248, 1991.

[18] J. Ren, Z. Cheng, and Y. Chen, "Existence results of periodic solutions for third-order nonlinear singular differential equation," Mathematische Nachrichten, vol. 286, no. 10, pp. 1022-1042, 2013.

[19] J. Sun and Y. Liu, "Multiple positive solutions of singular third-order periodic boundary value problem," Acta Mathematica Scientia, vol. 25, no. 1, pp. 81-88, 2005.

[20] H. Yu and M. Pei, "Solvability of a nonlinear third-order periodic boundary value problem," Applied Mathematics Letters, vol. 23, no. 8, pp. 892-896, 2010.

[21] X. Zhang, J. Jiang, Y. Wu, and Y. Cui, "Existence and asymptotic properties of solutions for a nonlinear Schrödinger elliptic equation from geophysical fluid flows," Applied Mathematics Letters, vol. 90, pp. 229-237, 2019.

[22] Y. Zhang, B. Liu, Y. Zhu, and M. Pei, "Existence and multiplicity of positive periodic solutions of a class of nonlinear third-order differential equation," Journal of Beihua University(Natural Science), vol. 20, pp. 299-303, 2019.

[23] P. Kelevedjiev and T. Todorov, "Existence of solutions of nonlinear third-order two-point boundary value problems," 
Electronic Journal of Qualitative Theory of Differential Equations, vol. 2019, no. 23, pp. 1-15, 2019.

[24] A. Granas, R. B. Guenther, and J. W. Lee, "Nonlinear boundary value problems for ordinary differential equations,"

Dissertationes Mathematicae, vol. 244, 1985. 